\title{
Effect of current sweep reversal on the magnetic field stability for a Bi-2223 superconducting solenoid
}

\author{
Y. Yanagisawa ${ }^{1,2}$, H. Nakagome ${ }^{2}$, Y. Koyama ${ }^{1,3}$, R. Hu${ }^{1,3}$, T. Takao ${ }^{3}$, M. Hamada ${ }^{4}$, \\ T. Kiyoshi ${ }^{5}$, M. Takahashi, ${ }^{1,6}$, and H. Maeda ${ }^{1,6}$
}
${ }^{1}$ Systems and Structural Biology Center, RIKEN, Yokohama, 230-0045, Japan
${ }^{2}$ Graduate School of Engineering, Chiba University, Chiba, 236-8522, Japan
${ }^{3}$ Sophia University, Yotsuya, Tokyo, 102-8554, Japan
${ }^{4}$ Kobe Steel, Ltd., Kobe, 651-2271, Japan
${ }^{5}$ Superconducting Materials Center, National Institute for Materials Science, Tsukuba, 305-0003, Japan

${ }^{6}$ Graduate School of Yokohama City University, Yokohama, 230-0045, Japan

Corresponding author:

Name: Hideaki Maeda

Phone: +81-045-508-7211

Fax: $+81-045-508-7360$

E-mail address: maeda@jota.gsc.riken.jp

(C) 2009. This manuscript version is made available under the Elsevier user license http://www.elsevier.com/open-access/userlicense/1.0/ 


\begin{abstract}
The effect of current sweep reversal on the temporal drift in magnetic field intensity for a Bi-2223 solenoid was investigated by experiment and using numerical simulation. Current sweep reversal, by as small as $1 \%$ of the peak current, was found to stabilize the drift in magnetic field intensity for a $\mathrm{Bi}-2223$ tape solenoid. The field drift was due to flux creep in the Bi-2223 tape and the current sweep reversal formed a barrier for flux entrance at the upper and lower surface of the conductor, preventing flux creep. With a current reversal of several $\%$ of the peak current, the barrier formation extended over half of the solenoid and the magnetic field intensity became constant with time. The current sweep reversal technique should prove useful to stabilize an ultra-high field low/high-temperature superconducting nuclear magnetic resonance magnet operated at frequencies (field intensities) beyond $1 \mathrm{GHz}(23.5 \mathrm{~T})$.
\end{abstract}


PACS

75.60. Ej

76.60. Es

84.71.Ba

87.64. $\mathrm{Kj}$

\section{Key words}

High temperature superconducting NMR magnet, Current sweep reversal,

Magnetic field stabilization, Screening current 


\section{Introduction}

In previous work [1], the authors have shown that the central magnetic field for $\mathrm{a}(\mathrm{Bi}, \mathrm{Pb})_{2} \mathrm{Sr}_{2} \mathrm{Ca}_{2} \mathrm{Cu}_{3} \mathrm{O}_{\mathrm{x}}(\mathrm{Bi}-2223)$ high temperature superconducting (HTS) coil exhibits hysteresis and a drift of its intensity with time. Both of these effects are caused by a screening current-induced magnetic field, and its relaxation has been shown to be governed by flux creep [1]. Temporal magnetic field drift is a serious problem for ultra-high field, low/high-temperature superconducting (LTS/HTS) nuclear magnetic resonance (NMR) spectrometer [2-4], making long term NMR measurement over days impossible. The purpose of this paper is to investigate the effect of current sweep reversal on the magnetic field drift for a $\mathrm{Bi}-2223$ solenoid, using both experiment and numerical simulation. Similar phenomena have been reported for magnetization of HTS crystals in an external magnetic field [5,6]. However, no such investigation has been made on an HTS coil.

\section{Numerical Simulation method}

In numerical simulation, a solenoid is modeled as a set of superconducting concentric coil-rings as seen in Figure 1 (a); each coil-ring corresponds to one turn, and is numbered as $\# 1$ to $\# M$, where $M$ is the number of turns for the solenoid. Considering 
the large aspect ratio of the Bi-2223 tape as shown in Fig. 1(b), we only calculate the azimuthal component of the screening current induced by the radial magnetic field $B_{r_{m}}$. Firstly, the motion of the screening current density in a Bi-2223 tape by arbitrary current sweep is numerically simulated using a model for a thin superconductor strip in a perpendicular magnetic field proposed by Brandt [7,8] and Yazawa et al. [9] The vertical width of the Bi-2223 multi-filamentary composite $2 a$ seen in Fig. 1(b) is discretized into $2 N$ points, while its thickness $d$ is not discretized.

In this paper, we estimate a time-dependent screening current density distribution in the Bi-2223 tape; the screening current density is the current density over the thickness of the Bi-2223 multi filamentary composite of the tape. Considering the symmetry of the current density $J(-y)=-J(y)$, we only calculate over $y \geq 0$. The discrete form of the equation of motion of the screening current density at the $i$ th discretized point in the $m$ th coil-ring, $J_{m, i}$, is given by [7-9]

$$
\begin{aligned}
& J_{m, i}(t+\Delta t)=J_{m, i}(t)+\frac{2 \pi \Delta t}{\mu_{0} d} \sum_{j=1}^{N} K_{i j}^{-1}\left[E_{m, j}(t)-y_{j} \dot{B}_{r m}(t)\right] \\
& (m=1, \ldots, M ; i=1, \ldots, N)
\end{aligned}
$$

where $i$ is a discretized field point, $j$ is a discretized source point, $t$ is time, $\mu_{0}$ is the magnetic permeability of vacuum, $K_{i j}$ is the integral kernel with geometrical information of the $\mathrm{Bi}-2223$ tape (which is a $N \times N$ matrix connecting a field point $y_{i}$ with a source 
point $y_{j}$ [7-9] ), $E_{m, j}$ is the electric field at the $j$ th source point in the $m$ th coil-ring, $y_{j}$ is the vertical coordinate of the $j$ th source point, and $B_{r_{m}}$ is the radial magnetic field for the $m$ th coil-ring. Here, $B_{r_{m}}$ is the sum of the radial magnetic fields generated by the other coil-rings. $B_{r_{m}}$ is calculated using elliptic integrals of the first and second kind [10]. As the ratio of the coil current to the conductor critical current in the solenoid is as small as $<0.3$, we ignore the self-field effect due to the transport current in the Bi-2223 tape $[9,10]$.

The normal transition for an HTS is given by the power law as [8]

$$
E_{m, j}=E_{c}\left(\frac{J_{m, j}}{J_{c m}}\right)^{n} \quad(m=1, \ldots, M ; j=1, \ldots, N)
$$

where $J_{c_{m}}$ is the overall critical current density in the Bi-2223 multi-filamentary composite of the tape for the $m$ th coil-ring, $E_{c}$ is the longitudinal electric field at $J_{c_{m}}$, and $n$ is the index number of the $\mathrm{Bi}-2223$ tape. The power law assumes a logarithmic current dependence of the activation energy and thermal activation processes such as flux creep.[8] The field dependence of the $J_{c_{m}}$ is assumed to be [11]

$$
\begin{aligned}
& J_{c m / /}=J_{c 0} \frac{B_{0 / /}}{B_{z m}+B_{0 / /}} \\
& J_{c m \perp}=J_{c 0} \frac{B_{0 \perp}}{B_{r m}+B_{0 \perp}} \quad(m=1, \ldots, M)
\end{aligned}
$$

where $J_{c m / /}$ and $J_{c m \perp}$ are the parallel-field overall critical current density and the 
perpendicular-field overall critical current density in the Bi-2223 multi-filamentary composite respectively, $J_{c 0}$ is the zero-field overall critical current density in the Bi-2223 multi-filamentary composite, and $B_{z_{m}}$ and $B_{r_{m}}$ are the axial and radial magnetic fields on the $m$ th coil-ring, respectively. The values of $B_{0 / /}$ and $B_{0 \perp}$ are determined based on the $J_{c}-B$ characteristics of the Bi-2223 tape used in this experiment. $M \times N$ sets of Eq. (1) are simultaneously solved for $J_{m, i}(m=1, \ldots, M ; i=1, \ldots, N)$ for each time step; for each calculation step, $J_{m, i}$ are recalculated on all coil-rings based on Eq. (2) and Eq. (3). The $J_{c m / /}$ value is compared with the $J_{c m \perp}$ value at each time step for individual coil-rings and the smaller value is chosen for $J_{c_{m}}$. Secondly, the screening current-induced magnetic fields are calculated using elliptic integrals of the first and second kind, based on the screening current density in each of the coil-rings.

The values of $a, d, E_{c}, J_{c 0}, B_{0 / /}, B_{0 \perp}$ and $n$ used in the numerical simulation are 2.0 $\mathrm{mm}, 0.2 \mathrm{~mm}, 1.0 \times 10^{-4} \mathrm{~V} / \mathrm{m}, 6.25 \times 10^{8} \mathrm{~A} / \mathrm{m}^{2}, 5.0 \mathrm{~T}, 0.8 \mathrm{~T}$, and 39 , respectively. The $n$ index value of 39 corresponds to the pinning barrier height of $14 \mathrm{meV}[8]$

\section{Experimental procedure}

A Bi-2223 solenoid was made by Kobe Steel, Ltd. and JASTEC, Inc. for the innermost coil of a $500 \mathrm{MHz}(11.75 \mathrm{~T}) \mathrm{NMR}$ spectrometer [12]. The Bi-2223 tape was 
$4.55 \mathrm{~mm}$ in width and $0.36 \mathrm{~mm}$ in thickness with copper alloy reinforcement sheets. The solenoid was $81 \mathrm{~mm}$ in inner diameter, $121 \mathrm{~mm}$ in outer diameter, and $375 \mathrm{~mm}$ in length. The solenoid develops a central magnetic field of $1.8 \mathrm{~T}$ at a coil current of 144A. It was originally made to combine with a low temperature superconducting (LTS) coil to generate $11.75 \mathrm{~T}$, corresponding to $500 \mathrm{MHz}$ spectrometer operation; however, only the HTS coil was charged in the present experiment. A 4 Kelvin Gifford-McMahon cycle cryocooler was installed on top of the cryostat, enabling long-term magnet operation over several months without refilling liquid helium [13]. The axial components of the magnetic field at the coil center, $z=0 \mathrm{~mm}$, and at the coil end, $z=186 \mathrm{~mm}$, were measured by Hall sensors (BH200, F.W. Bell).

\section{Results and discussion}

Figure 2 shows the time-dependent component of the magnetic field drift after reversal of the current sweep; the data shown in Fig. 2(a) were measured at the coil center and those of Fig. 2(b) at the coil end. After each measurement, the current was reduced to $0 \mathrm{~A}$. For the first current ramp (black line) from $0 \mathrm{~A}$ to $144 \mathrm{~A}$, the magnetic field at 144A showed a positive drift at the coil center [1], while it exhibited a negative drift at the coil end. The screening current-induced magnetic field at the coil center was 
of opposite polarity to that induced by the coil current, and therefore the magnetic field intensity showed a positive drift with time. On the other hand, the magnetic field at the coil end was of the same polarity as the coil magnetic field, and the magnetic field intensity showed a negative drift with time. The numerical simulation results shown in Fig. 2(c) for the coil center and Fig. 2(d) for the coil end agree with the experimental results. For the second current cycle (blue line) from $0 \mathrm{~A}$ to $144 \mathrm{~A}$ and then to $142.6 \mathrm{~A}$, the drift slope of the magnetic field intensity at 142.6A became much smaller than the first current ramp; the same result was obtained by numerical simulation. The effect is dramatic for the third cycle (red line) at 136.8A, where the magnetic field intensity became nearly constant with time. Thus, it is demonstrated that current sweep reversal by as small as $1 \%$ of the peak current stabilizes the drift of the magnetic field intensity. For the fourth current cycle (green line) from $0 \mathrm{~A}$ to $144 \mathrm{~A}$ to $0 \mathrm{~A}$, the magnetic field intensity in the coil center at $0 \mathrm{~A}$ showed a negative drift with time [1] and for the fifth current cycle (orange line) from $0 \mathrm{~A}$ to $144 \mathrm{~A}$ to $-144 \mathrm{~A}$, the drift at $-144 \mathrm{~A}$ was nearly the same as that of the first cycle, while its polarity was reversed.

Figure 3 shows the screening current density profiles in Bi-2223 tapes which were simulated for the five consecutive current cycles shown in Fig. 2 at locations A, B, and $\mathrm{C}$ in the solenoid (see the inset of Fig. 3). The initial profile achieved just after 
reaching a set current is indicated by red closed circles, while the profile at $t=10 \mathrm{~h}$ is indicated by blue open circles. The vertical axis, screening current density, is normalized to the zero-field overall critical current density, $J_{c 0}$, while the horizontal axis, the vertical location within the $\mathrm{Bi}-2223$ tape, is normalized to the half width of the Bi-2223 multi-filamentary composite, $a$. In the case of the first current ramp at 144A, the screening current partially penetrates at point $\mathrm{B}$ while it fully penetrates at point $\mathrm{C}$ as indicated by the dashed arrows in Fig. 3. At $t=10 \mathrm{~h}$, their intensities are reduced and tend to become horizontal, corresponding to the decrease in magnetic field gradient and approaching the linear profile due to flux creep. For the second current cycle at 142.6A, the screening current densities at the upper and lower ends of the tape are nearly zero at point $\mathrm{C}$, which is equivalent to the zero gradient of the magnetic field; the screening current acts as a barrier against entrance of the magnetic flux, and thus flux creep in the Bi-2223 tape stops. The barrier formation is limited to the vertical ends of the solenoid where the radial field is highest. For the third current cycle at $136.8 \mathrm{~A}$, the screening current densities at the upper and lower ends of the Bi-2223 tape are nearly zero for point $\mathrm{B}$ and become negative at point $\mathrm{C}$; i.e. magnetic flux barriers with zero-gradient or negative gradient of the magnetic field are formed over more than half of the coil volume and therefore the magnetic field is fully stabilized as seen in Fig. 2. For the 
fourth current cycle at $0 \mathrm{~A}$, the drop in current density at the Bi-2223 tape boundaries nearly vanishes; the profile resembles the first cycle and therefore flux creep appears again and the magnetic field intensity drifts with time in the reverse direction. Finally, for the fifth cycle at $-144 \mathrm{~A}$, the current density profile completely returns to that of the first cycle, while its polarity is reversed.

Current sweep reversal by a small amount is widely used to stabilize the persistent magnetic field decay of LTS NMR magnets $[14,15]$. For a LTS NMR coil, the central magnetic field intensity decreases with time after the coil charge; if the current sweep is reversed by a small amount $(<1 \%)$, the magnetic field is sufficiently stabilized. If the current is reversed too much, the magnetic field begins to increase with time. These behaviors resemble those of the Bi-2223 coil seen in Fig. 2(a) and they may be due to flux creep. Differences in drift direction between LTS NMR magnets and the Bi-2223 HTS solenoid are due to the geometrical distribution of the magnetization; i.e., for LTS NMR magnets, the irreversible magnetization on superconducting filaments is uniformly distributed in the coil, while for the Bi-2223 HTS coil, the screening current concentrates at the coil ends. Thus, it is suggested that a current sweep reversal by $<1 \%$ of the peak current is effective to stabilize the ultra-high magnetic field LTS/HTS NMR magnet operated at frequencies beyond $1 \mathrm{GHz}$ (field strengths $>23.5 \mathrm{~T}$ ) [2-4], as this 
simultaneously stabilizes both LTS back up coils and an HTS inner coil.

\section{Conclusions}

Current sweep reversal, by as small as $1 \%$ of the peak current, stabilizes the drift in magnetic field intensity for a Bi-2223 tape solenoid. The field drift is due to flux creep in the Bi-2223 tape and the current sweep reversal forms a barrier for flux entrance, preventing flux creep. With a current reversal of several $\%$ of the peak current, the barrier formation extends over half of the solenoid and the magnetic field intensity becomes constant with time. The current sweep reversal technique is useful to stabilize an ultra-high field LTS/HTS NMR magnet operated at frequencies beyond $1 \mathrm{GHz}$ (magnetic fields greater than 23.5T).

This work was supported by SENTAN, JST in Japan. 


\section{References}

[1] Y. Koyama, T. Takao, Y. Yanagisawa, H. Nakagome, M. Hamada, T. Kiyoshi, M. Takahashi, and H. Maeda, Towards beyond 1GHz NMR: mechanism of the long term drift of screening current-induced magnetic field in a Bi-2223 coil, Physica $C$ 469 (2009) 694.

[2] Y. Yanagisawa, H. Nakagome, M. Hosono, M. Hamada, T. Kiyoshi, F. Hobo, M.

Takahashi, T. Yamazaki, and H. Maeda, Towards beyond-1GHz solution NMR:

internal ${ }^{2} \mathrm{H}$ lock operation in an external current mode, J. Mag. Res. 192 (2008) 329.

[3] T. Kiyoshi, A. Otsuka, S. Choi, S. Matsumoto, K. Zaitsu, T. Hase, M. Hamada, M. Hosono, M. Takahashi, T. Yamazaki, and H. Maeda, NMR Upgrading Project towards 1.05GHz, IEEE Trans. Appl. Supercond. 18 (2008) 860.

[4] S. Hahn, J. Bascunan, W. Kim, E. S. Bobrov, H. Lee, and Y. Iwasa, Operation and performance analyses of 350 and $700 \mathrm{MHz}$ low-/high-temperature superconductor nuclear magnetic resonance magnets: A march toward operating frequencies above 1GHz, J. Appl. Phys. 105 (2009) 024501.

[5] K. Kwasnitza and C. Widner, Strong magnetic history dependence of magnetic relaxation in high- $\mathrm{T}_{\mathrm{c}}$ superconductors, Physica C 184 (1991) 341.

[6] K. Kwasnitza and Ch. Widmer, On the reduction of flux-creep in superconducting 
accelerator magnets, IEEE Trans. Mag. 27 (1991) 2515.

[7] E. H. Brandt, Thin superconductors in a perpendicular magnetic ac field: General formulation and strip geometry, Phy. Rev. B 49 (1994) 9024.

[8] E. H. Brandt, Superconductor of finite thickness in a perpendicular magnetic field: Strips and and slabs, Phys. Rev. B 54 (1996) 4246.

[9] T. Yazawa, J. J. Rabbers, B. ten Haken, H. H. J. ten Kate, H. Maeda, AC loss analysis on high-temperature superconductors with finite thickness and arbitrary magnetic field dependent voltage-current relation, J. Appl. Phys. 84 (1998) 5652.

[10] N. Amemiya and K. Akachi, Magnetic field generated by shielding current in high $T_{\mathrm{c}}$ superconducting coils for NMR magnets, Supercond. Sci. Technol. 21 (2008) 095001.

[11] P. W. Anderson, Theory of flux creep in hard superconductors, Phys. Lev. Lett. 9 (1962) 309 .

[12] Y. Yanagisawa, H. Nakagome, K. Tennmei, M. Hamada, M. Yoshikawa, A. Otsuka, M. Hosono, K. Tsukasa, M. Takahashi, T. Yamazaki, and H. Maeda, Towards an NMR spectrometer beyond $1 \mathrm{GHz}$ : Operation of the $500 \mathrm{MHz}$ LTS/HTS NMR, $50^{\text {th }}$ experimental nuclear magnetic resonance conference, (2009) WOE 5.

[13] A. Otsuka, T. Kiyoshi, S. Matsumoto, K. Kominato, and M. Takeda, Filed 
stability of a $600 \mathrm{MHz}$ NMR magnet in the driven-mode operation, IEEE Trans. Appl. Supercond. 18 (2008) 852.

[14] G. Luderer and P. Dullenkopf, Influence of flux penetration on magnetic field stability, Cryogenics, (1976) 42.

[15] L. Cesnak and J. Kokavec, Magnetic field stability of superconducting magnets, Cryogenics, February, (1977) 107. 


\section{Figure captions}

Figure 1: Numerical simulation model for a time-dependent screening current-induced magnetic field. (a) Screening current is induced in the Bi-2223 tape by the radial magnetic field. (b) Motion of the current density is numerically simulated based on the superconducting thin strip model.

Figure 2: Effect of current sweep reversal on magnetic field drift with time. Experimental results (a) at the coil center and (b) at the coil end. Simulation results (c) at the coil center and (d) at the coil end.

Figure 3: The simulated screening current density profiles at three locations in the Bi-2223 solenoid. The vertical axis shows the screening current density normalized to the zero field overall critical current density, $J_{c 0}$, while the horizontal axis gives the vertical location in the Bi-2223 tape normalized to the half of the Bi-2223 multi filamentary composite width, $a$. Initial magnetic field profiles at $t=0 \mathrm{~h}$ and those at $t=$ $10 \mathrm{~h}$ are compared. Points $\mathrm{A}, \mathrm{B}$, and $\mathrm{C}$ in the upper inset show the locations of the screening current density profiles in the solenoid. 

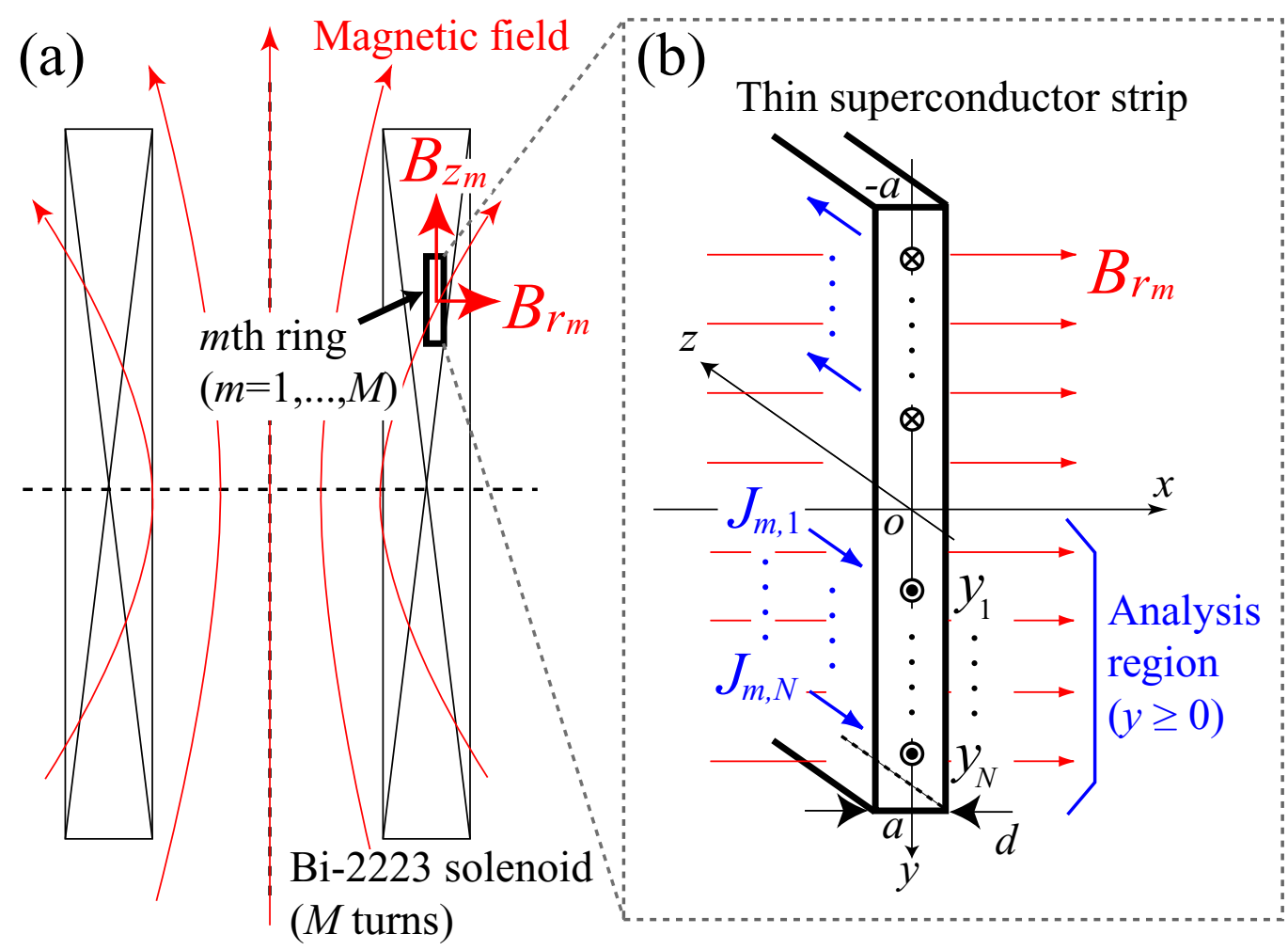
Experiment
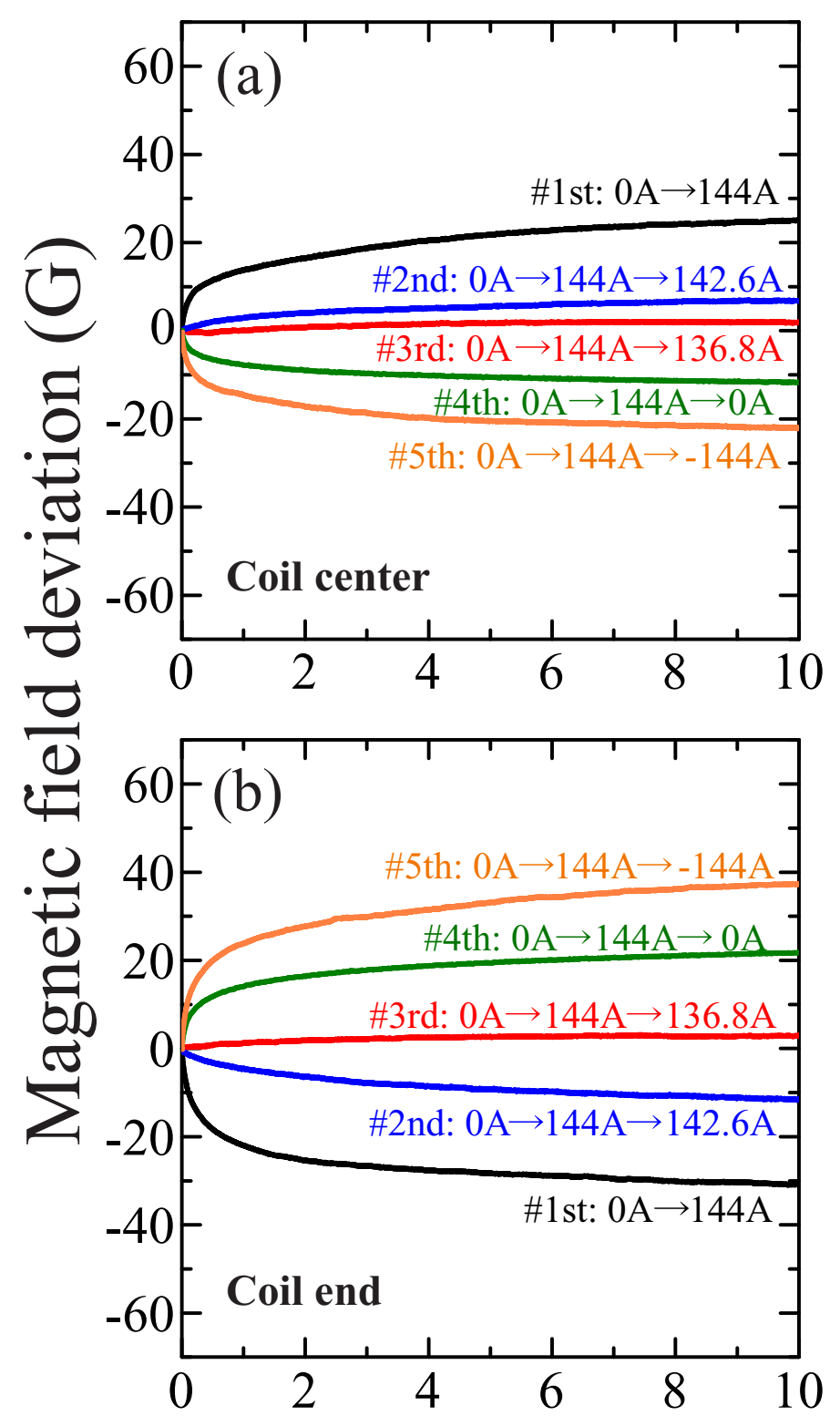

Simulation
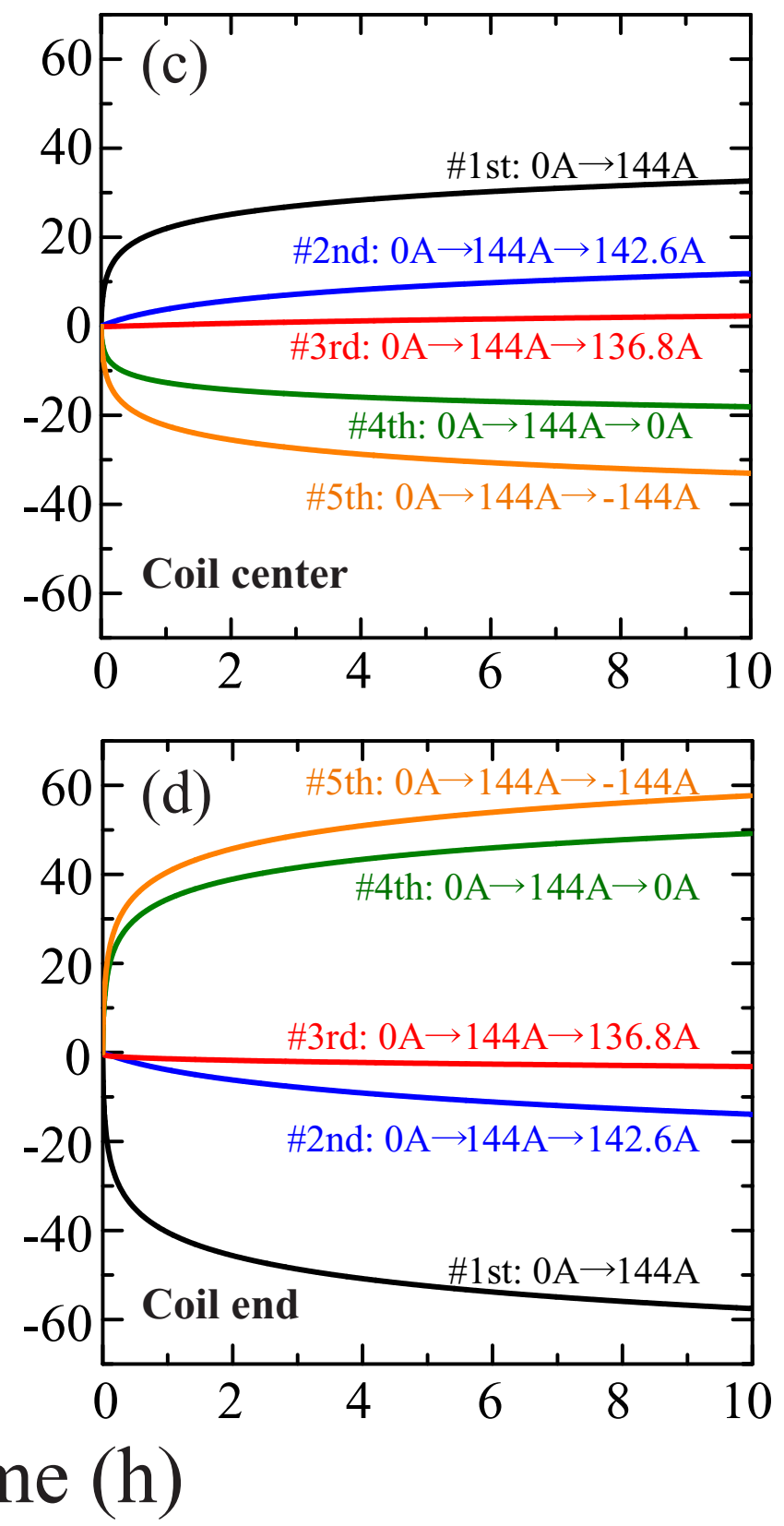


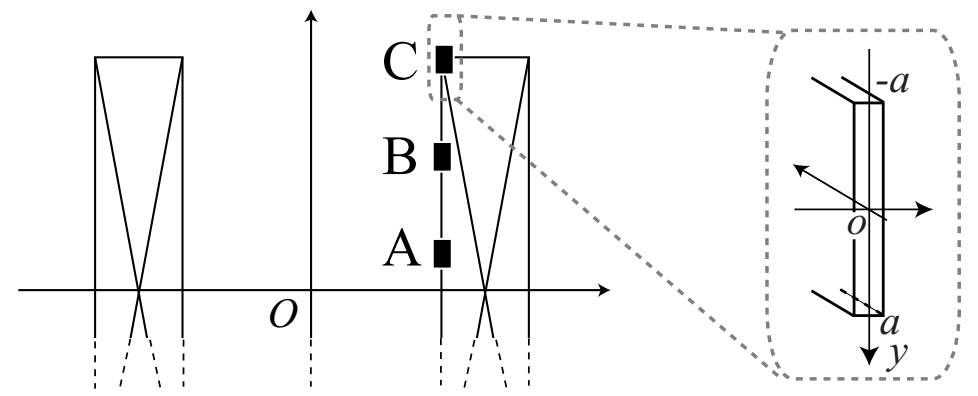

$$
\begin{aligned}
& \multimap t=0 \mathrm{~h} \\
& -t=10 \mathrm{~h}
\end{aligned}
$$

1st current ramp 2nd current cycle 3rd current cyclel 4th current cycle 5th current cycle

$$
0 \mathrm{~A} \rightarrow 144 \mathrm{~A} \quad 0 \mathrm{~A} \rightarrow 144 \mathrm{~A} \quad 0 \mathrm{~A} \rightarrow 144 \mathrm{~A} \quad 0 \mathrm{~A} \rightarrow 144 \mathrm{~A} \quad 0 \mathrm{~A} \rightarrow 144 \mathrm{~A}
$$

$\mathrm{C}$
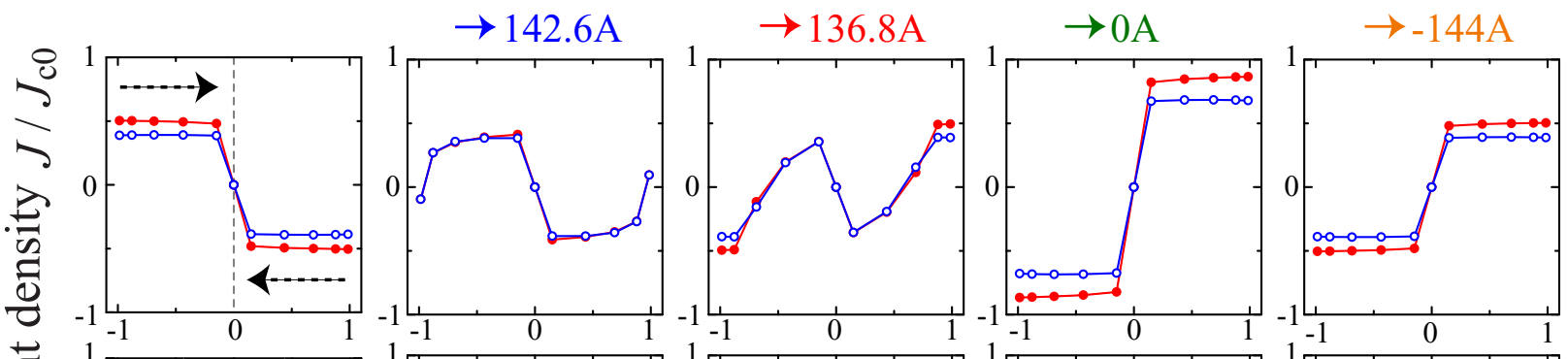

B
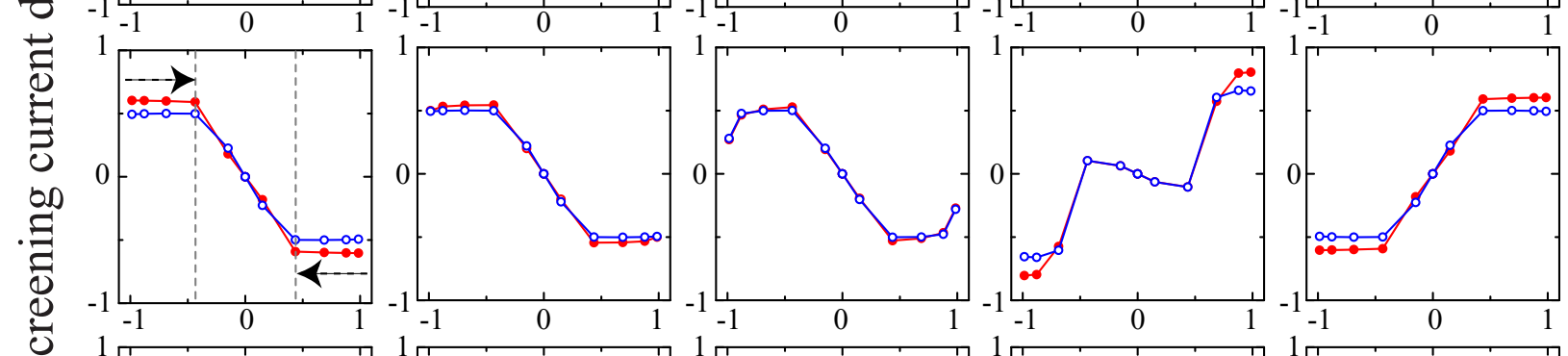

A
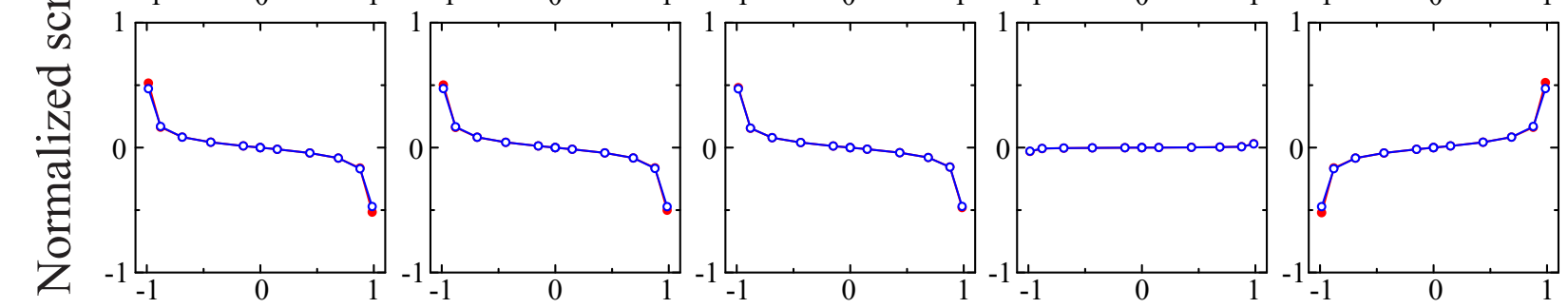

Normalized vertical position in the tape $y / a$ 